\title{
SYMMETRY REDUCTION AND NUMERICAL SOLUTION OF A THIRD-ORDER ODE FROM THIN FILM FLOW
}

\author{
E. Momoniat ${ }^{1}$ and F.M. Mahomed ${ }^{2}$ \\ Centre for Differential Equations, Continuum Mechanics and Applications \\ School of Computational and Applied Mathematics, \\ University of the Witwatersrand, Johannesburg, \\ Private Bag 3, Wits 2050, \\ ${ }^{1}$ Ebrahim.Momoniat@wits.ac.za, ${ }^{2}$ Fazal.Mahomed@wits.ac.za
}

\begin{abstract}
A new approach to solving high-order ordinary differential equations numerically is presented. Instead of the usual approach of writing a high-order ordinary differential equation as a system of first-order ordinary differential equations, we write the high-order ordinary differential equation in terms of its differential invariants. The third-order ordinary differential equation $y^{\prime \prime \prime}=y^{-k}$ for constant $k$ is used to illustrate this approach for the cases $k=2$ and $k=3$.
\end{abstract}

Keywords- Thin film; third-order ODE; symmetry reduction.

\section{INTRODUCTION}

We consider the ordinary differential equation (ODE)

$$
y^{\prime \prime \prime}=y^{-k}, \quad k=\text { constant }
$$

solved subject to the initial conditions $(\alpha, \beta, \gamma$ and $\lambda$ are constants)

$$
y(\lambda)=\alpha, \quad y^{\prime}(\lambda)=\beta, \quad y^{\prime \prime}(\lambda)=\gamma
$$

Analytical solutions to the third-order ODE (1) are given in general in the handbook of Polyanin and Zaitsev [17] for the cases $k=0,7 / 2,5 / 2,4 / 3,7 / 6,2,1 / 2$ and 5/4. The ordinary differential equation for the case $k=2$ has been derived by Tanner [18] to investigate the motion of the contact line for a thin oil drop spreading on a horizontal surface. A review of thin film flow has been presented by Myers [14] in which some third-order ODEs occuring in thin film flow are discussed. The interested reader is also referred to Tuck and Schwartz [19] and the references therein. Analytical solutions admitted by the case $k=2$ has also recently been investigated by Duffy and Wilson [4]. The analytical solution for the case $k=2$ was initially presented by Ford [5]. The case $k=2$, amongst others, has been the subject of intense numerical investigation by Tuck and Schwartz [19]. Existence and uniqueness 
of solutions admitted by (1) are discussed in Troy [21] and Bernis [1]. Momoniat [11] has investigated autonomous integrals of (1). A numerical investigation of boundary value problems associated with (1) have recently been considered by Momoniat [12].

In this paper we consider the equation (1) for arbitrary $k$. We demonstrate our proposed algorithm by considering the cases $k=2$ and $k=3$. The case $k=3$, which has not been investigated before for analytical solutions, can be used to model the draining down of a dry wall of a non-Newtonian thin film. Alternatively, it can represent the steady-state solution to the nonlinear partial differential equation

$$
\frac{\partial h}{\partial t}=\frac{\partial}{\partial x}\left(h^{3} \frac{\partial^{3} h}{\partial x^{3}}\right),
$$

which describes the surface tension driven spreading of a thin viscous film (see e.g. Myers [14]). The results obtained here are general and can be applied to high-order initial value problems that admit Lie point symmetries.

\section{SYMMETRY REDUCTION}

\subsection{Point Symmetries}

We apply the Lie group method to the ODE (1) in general before considering the specific case $k=3$ which has not been investigated before analytically.

The interested reader is referred to the books by Bluman and Kumei [3], Ibragimov [9], Olver [15] and Ovsiannikov [16] on the Lie group method for solving differential equations.

The operator

$$
X=\xi(x, y) \partial_{x}+\eta(x, y) \partial_{y}
$$

generates a point symmetry of (1) if the determining equation

$$
\left.X^{[3]}\left(y^{\prime \prime \prime}-y^{-k}\right)\right|_{y^{\prime \prime \prime}=y^{-k}}=0
$$

holds, where $\left.\right|_{y^{\prime \prime \prime}=y^{-k}}$ refers to an evaluation on equation (1). Also, the third extension or prolongation of the generator $X$ is given by

$$
X^{[3]}=X+\zeta^{1} \partial_{y^{\prime}}+\zeta^{2} \partial_{y^{\prime \prime}}+\zeta^{3} \partial_{y^{\prime \prime \prime}}
$$

where

$$
\zeta^{j}\left(x, y, y^{\prime}, \ldots, y^{(j)}\right)=\frac{d \zeta^{j-1}}{d x}-y^{(j)} \frac{d \xi}{d x}, \quad j=1,2,3
$$


with $y^{(j)}=d^{j} y / d x^{j}, \zeta^{0}=\eta(x, y)$ and $d / d x$ is the operator of total differentiation given by

$$
\frac{d}{d x}=\frac{\partial}{\partial x}+y^{\prime} \frac{\partial}{\partial y}+y^{\prime \prime} \frac{\partial}{\partial y^{\prime}}+\ldots
$$

By use of the approach of Lie one can calculate $\xi(x, y)$ and $\eta(x, y)$ systematically. The determining equation (5) can clearly be written as

$$
\left.\zeta^{3}\right|_{y^{\prime \prime \prime}=y^{-k}}+k \eta y^{-k-1}=0,
$$

where $\zeta^{3}$ is given in (7). The expansion of (9) by use of (7) (and its expansion in terms of $\xi$ and $\eta$ ) results in

$$
\begin{gathered}
\eta_{x x x}+\left(3 \eta_{x x y}-\xi_{x x x}\right) y^{\prime}+3\left(\eta_{x y y}-\xi_{x x y}\right) y^{\prime 2}+\left(\eta_{y y y}-3 \xi_{x y y}\right) y^{\prime 3} \\
-\xi_{y y y} y^{\prime 4}+3\left(\eta_{x y}-\xi_{x x}\right) y^{\prime \prime}+3\left(\eta_{y y}-3 \xi_{x y}\right) y^{\prime} y^{\prime \prime}-6 \xi_{y y} y^{\prime 2} y^{\prime \prime} \\
-3 \xi_{y} y^{\prime \prime 2}+\left(\eta_{y}-3 \xi_{x}\right) y^{-k}-4 \xi_{y} y^{-k} y^{\prime}+k \eta y^{-k-1}=0 .
\end{gathered}
$$

The determining equation (10) can be separated by coefficients of derivatives of $y$. Solutions of the resultant system of linear partial differential equations are

$$
\xi=a_{0}+a_{1}(k+1) x, \quad \eta=3 a_{1} y,
$$

where $a_{0}$ and $a_{1}$ are arbitrary constants. Setting firstly $a_{1}=0$ and then $a_{0}=0$ we obtain the two Lie point symmetry generators

$$
X_{1}=\partial_{x}, \quad X_{2}=(k+1) x \partial_{x}+3 y \partial_{y}
$$

Lie point symmetries admitted by differential equations are easily obtainable by the use of computer algebra packages (see e.g., Head [7], Sherring et al. [20] and Baumann [2]).

\subsection{Group Invariant Solutions}

Here we deduce a group invariant solution admitted by (1). We show that the initial conditions (2) are satisfied only if they are in terms of $\alpha$.

We consider a linear combination of the Lie point symmetries (12) of the form

$$
X=a_{1} X_{1}+a_{2} X_{2}
$$

where $a_{1}$ and $a_{2}$ are constants to be determined. We determine a group invariant solution $y=\Gamma(x)$ admitted by (1) by solving the first-order ODE obtained from

$$
\left.X(y-\Gamma(x))\right|_{y=\Gamma(x)}=0 .
$$


The solution of (14) yields

$$
y(x)=a_{3}\left(a_{1}+a_{2}(k+1) x\right)^{\frac{3}{k+1}}, \quad k \neq-1,
$$

where $a_{3}$ is a constant of integration. The substitution of (15) into (1) gives

$$
a_{3}=\left(3 a_{2}^{3}(k-2)(2 k-1)\right)^{-\frac{1}{k+1}} .
$$

Without loss of generality we can choose $a_{2}=1$ otherwise we get the trivial solution. We determine the constant $a_{1}$ by imposition of the initial condition

$$
y(\lambda)=\alpha
$$

to find that

$$
a_{1}=\alpha^{\frac{k+1}{3}}(3(k-2)(2 k-1))^{\frac{1}{3}}-(k+1) \lambda .
$$

We then calculate the values for $\beta$ and $\gamma$ in terms of $\alpha$. The invocation of (2) on (15) gives rise to

$$
\beta=\frac{3^{\frac{2}{3}} \alpha^{\frac{2-k}{3}}}{((k-2)(2 k-1))^{\frac{1}{3}}}, \quad \gamma=-\frac{3^{\frac{1}{3}} \alpha^{\frac{1-2 k}{3}}(k-2)^{\frac{1}{3}}}{(2 k-1)^{\frac{2}{3}}}, \quad k \neq \frac{1}{2}, 2 .
$$

For $k=-1$, from (14) we obtain $y=a_{3} \exp \left(3 a_{2} x / a_{1}\right)$, where $a_{3}$ is a constant, which does not give rise to an invariant solution. Specifically, when $k=3$ we find that

$$
y(x)=15^{-\frac{1}{4}}\left(15^{\frac{1}{3}} \alpha^{\frac{4}{3}}-4 \lambda+4 x\right)^{\frac{3}{4}} .
$$

To prevent the solution (20) from becoming imaginary, the following condition needs to be maintained

$$
x \geq \frac{4 \lambda-15^{\frac{1}{3}} \alpha^{\frac{4}{3}}}{4} .
$$

From (19), for $k=3$, we find that

$$
\beta=\frac{3^{\frac{2}{3}}}{5^{\frac{1}{3}} \alpha^{\frac{1}{3}}}, \quad \gamma=-\frac{3^{\frac{1}{3}}}{5^{\frac{2}{3}} \alpha^{\frac{5}{3}}} .
$$

The group invariant solution (15) is not valid for the cases $k=2$ and $k=1 / 2$. For both the cases $k=2$ and $k=1 / 2$ we are unable to determine group invariant solutions. In the case $k=2$ the exponent in (15) becomes one. On substitution into (1) we are unable to satisfy the right-hand side without the solution becoming singular. The same holds for $k=1 / 2$ where the exponent in (15) becomes two. 


\subsection{Group Reduction}

The algebra formed by the Lie point symmetries is two-dimensional since

$$
\left[X_{1}, X_{2}\right]=(k+1) X_{1}
$$

Hence we firstly perform a reduction on (1) using $X_{1}$. Then $X_{2}$ is a Lie point symmetry generator of the reduced equation. An invariant corresponding to $X_{1}$ is given by

$$
y^{\prime}=v
$$

We can use this invariant as follows

$$
\begin{aligned}
& \frac{d^{2} y}{d x^{2}}=y^{\prime} \frac{d y^{\prime}}{d y}=v \frac{d v}{d y} . \\
& \frac{d^{3} y}{d x^{3}}=y^{\prime} \frac{d y^{\prime \prime}}{d y}=v \frac{d}{d y}\left(v \frac{d v}{d y}\right) .
\end{aligned}
$$

The initial conditions (2) then imply that

$$
v(\alpha)=\beta, \quad v^{\prime}(\alpha)=\gamma / \beta .
$$

Equation (1) can be reduced to

$$
\frac{d^{2} v}{d y^{2}} v^{2}+v\left(\frac{d v}{d y}\right)^{2}=y^{-k}
$$

The Lie point symmetry generator $X_{2}$ is admitted by (28) and is written in terms of the new variables as

$$
Y=3 y \partial_{y}+(2-k) v \partial_{v}
$$

The first prolongation of (29) is given by

$$
Y^{[1]}=Y-(k+1) v^{\prime} \partial_{v^{\prime}}
$$

Invariants corresponding to (30) are given by

$$
p=v y^{\frac{k-2}{3}}, \quad w=v^{\prime} v^{-\left(\frac{k+1}{k-2}\right)}, \quad k \neq 2 .
$$

The second-order ordinary differential equation (28) can then be reduced to the first-order ordinary differential equation

$$
\frac{d w}{d p}=\frac{\frac{1-2 k}{k-2} w^{2} p^{\frac{3}{k-2}}+p^{\frac{3-3 k}{k-2}}}{w p^{\frac{k+1}{k-2}}+\frac{k-2}{3} p}, \quad k \neq 2 .
$$


For the case $k=3$ the ODE (32) is solved subject to the initial conditions

$$
w\left(\alpha^{\frac{1}{3}} \beta\right)=\gamma \beta^{-5} \text {. }
$$

For the case $k=3$ from (31), (24) and (25) we have that

$$
y^{\prime \prime}=y^{\prime 5} w\left(y^{\prime} y^{\frac{1}{3}}\right) .
$$

The first-order reduction (32) is not valid for the case $k=2$. For this particular case the first prolongation of the Lie point symmetry generator (30) is

$$
Y^{[1]}=y \partial_{y}-v^{\prime} \partial_{v^{\prime}}
$$

Invariants corresponding to (35) are given by

$$
q=v, \quad z=v^{\prime} y
$$

Equation (28) for $k=2$ can then be written as the following first-order ODE in terms of the invariants $q$ and $h$ :

$$
\frac{d z}{d q}=1+\frac{1}{q^{2} z}-\frac{z}{q}
$$

The first-order ODE (37) is solved subject to

$$
z(\beta)=\alpha \gamma \beta^{-1}
$$

Then from (36), (24) and (25) we have that

$$
y^{\prime \prime}=y^{-1} y^{\prime} z\left(y^{\prime}\right)
$$

We utilise the above results in the next section.

\section{NUMERICAL STUDY}

We have reduced the third-order ODE $y^{\prime \prime \prime}=y^{-k}$ by successive reduction of order to a second-order ODE (28) and then to a first-order ODE (32) or (37). Unfortunately, for general $k$, (32) cannot be solved analytically. We can however use these reductions to determine an efficient way to solve (1) numerically. We focus on the cases $k=2$ and $k=3$.

A fourth-order Runge-Kutta method to solve the first-order ODE $y^{\prime}=f(x, y)$ gives values $y_{i}=y\left(x_{i}\right)$ where $x_{i}=\lambda+i h$ and $h$ is the step length. An initial value $y_{0}=y(0)$ is chosen with successive values determined by

$$
y_{i+1}=y_{i}+\frac{h}{6}\left(k_{1}+2 k_{2}+2 k_{3}+k_{4}\right)
$$


where

$$
\begin{gathered}
k_{1}=f(x, y), \quad k_{2}=f\left(x+\frac{h}{2}, y+\frac{h}{2} k_{1}\right), \quad k_{3}=f\left(x+\frac{h}{2}, y+\frac{h}{2} k_{2}\right), \\
k_{4}=f\left(x+h, y+h k_{3}\right) .
\end{gathered}
$$

We consider the initial conditions

$$
y(0)=y^{\prime}(0)=y^{\prime \prime}(0)=1
$$

i.e., we have taken

$$
\lambda=0, \quad \alpha=\beta=\gamma=1
$$

To use a fourth-order Runge-Kutta method we write (1) as a system of three first-order equations:

$$
y^{\prime}=p, \quad p^{\prime}=q, \quad q^{\prime}=y^{-k}
$$

where

$$
y(0)=1, \quad p(0)=1, \quad q(0)=1
$$

The results are displayed in Table 1 for the case $k=2$ and Table 2 for the case $k=3$.

From Duffy and Wilson [4] the exact solution for the case $y^{\prime \prime \prime}=y^{-2}$ is given in parametric form as

$$
\begin{aligned}
& x=2^{\frac{1}{3}} \pi \frac{A i(s) B i\left(s_{0}\right)-A i\left(s_{0}\right) B i(s)}{\left(a A i\left(s_{0}\right)+b B i\left(s_{0}\right)\right)(a A i(s)+b B i(s))}, \\
& y=(a A i(s)+b B i(s))^{-2},
\end{aligned}
$$

where $A i(s)$ and $B i(s)$ denote the Airy functions and $a, b$ and $s_{0}$ are constants to be determined. Imposing the initial conditions (42) we find that

$$
a=0.676482, \quad b=1.60629, \quad s_{0}=-0.39685 .
$$

The results for the exact solution are presented in Table 1 for a comparison.

The numerical solution for the cases $k=2$ and $k=3$ can be achieved in two ways. Firstly, the second-order ordinary differential equation (28) is solved numerically 
using a fourth-order Runge-Kutta method subject to (27). We do this by writing (28) as a system of two equations

$$
v^{\prime}=m, \quad m^{\prime}=\frac{y^{-k}-v m^{2}}{v^{2}} .
$$

The conditions (27) imply that

$$
v(1)=1, \quad m(1)=1
$$

We form a function $v(y)$ using cubic interpolation in MATHEMATICA [22]. The first-order ODE (24) is solved numerically using a fourth-order Runge-Kutta method. These results are displayed in Table 1 for $k=2$ and Table 2 for $k=3$ in the column labelled Method 1.

A second method which can be employed due to the double reduction of (1) is to solve the equivalent first-order ordinary differential equations. For the case $k=2$ we solve the first-order ODE (37) subject to (38) using the fourth-order Runge-Kutta method (41). The function $z=z(q)$ is formed via interpolation in MATHEMATICA [22]. The second-order ODE (39) is then solved using (41). The results using this second approach for $k=2$ are indicated in Table 1 under the column labelled Method 2.

For $k=3$ we solve firstly (32) subject to (33) using (41). We again interpolate the function $w(p)$ using MATHEMATICA [22]. The second-order ODE (34) is then solved using (41). These results are displayed in Table 2 in the column labelled Method 2.

\begin{tabular}{|c|c|c|c|c|}
\hline $\mathrm{x}$ & Exact Solution & RK4 & Method 1 & Method 2 \\
\hline 0.0 & 1.000000000 & 1.000000000 & 1.000000000 & 1.000000000 \\
0.2 & 1.221211030 & 1.221210005 & 1.221210004 & 1.221216123 \\
0.4 & 1.488834893 & 1.488834780 & 1.488834779 & 1.488884596 \\
0.6 & 1.807361404 & 1.807361398 & 1.807361397 & 1.807527232 \\
0.8 & 2.179819234 & 2.179819234 & 2.179819233 & 2.180203564 \\
1.0 & 2.608275822 & 2.608274868 & 2.608274867 & 2.609006355 \\
\hline
\end{tabular}

Table 1: Table comparing numerical values of the exact solution, a fourth-order Runge-Kutta method and our new approach at $x \in[0,0.2,0.4,0.6,0.8,1.0]$ taking $h=0.01$ and $k=2$ for the initial conditions $y(0)=y^{\prime}(0)=y^{\prime \prime}(0)=1$.

From Table 1 we observe that the numerical results using Method 1 are correct to 5 decimal places. The numerical results from Method 2 are only correct to 2 
decimal places. Applying the fourth-order Runge-Kutta method to (1) for $k=2$ also yields five decimal place accuracy.

\begin{tabular}{|c|c|c|c|}
\hline $\mathrm{x}$ & RK4 & Method 1 & Method 2 \\
\hline 0.0 & 1.000000000 & 1.000000000 & 1.000000000 \\
0.2 & 1.221155142 & 1.221155142 & 1.221162945 \\
0.4 & 1.488105284 & 1.488105284 & 1.488166464 \\
0.6 & 1.804262548 & 1.804262548 & 1.804459968 \\
0.8 & 2.171522798 & 2.171522797 & 2.171968706 \\
1.0 & 2.590958259 & 2.590958258 & 2.591788995 \\
\hline
\end{tabular}

Table 2: Table comparing numerical values of the numerical solution obtained using a fourth-order Runge-Kutta method and our new approach at $x \in$ $[0,0.2,0.4,0.6,0.8,1.0]$ taking $h=0.01$ and $k=3$ for the initial conditions $y(0)=y^{\prime}(0)=y^{\prime \prime}(0)=1$.

From Table 2 we observe that the numerical results using the fourth-order RungeKutta and Method 1 agree to 6 decimal places. Method 2 generates results correct to 2 decimal places. This is consistent with the results displayed in Table 1.

\section{CONCLUDING REMARKS}

The approach discussed in this paper is useful for validation of numerical results when no analytical results are known. The advantage in using this approach is that the same numerical algorithm is used, i.e. fourth-order Runge-Kutta. We note that for (1) we have obtained two validation approaches because of the double reduction effected by the occurence of two Lie point symmetries. The first approach, Method 1 , is the most accurate of the two. The reduced second-order ODE (28) has a nonlinearity at $y=0$ which is easily avoided. The second approach, Method 2, requires the solution of two highly nonlinear first-order ODEs (32) and (37).

The approach taken in this paper can easily be extended to the third-order ODEs $y^{\prime \prime \prime}=f(y)$ considered by Tuck and Schwartz [19] where

$$
\begin{aligned}
& f(y)=-1+y^{-2}, \\
& f(y)=-1+\left(1+\delta+\delta^{2}\right) y^{-2}-\left(\delta+\delta^{2}\right) y^{-3}, \\
& f(y)=y^{-2}-y^{-3} \\
& f(y)=y^{-2} .
\end{aligned}
$$


Typically, equations (51)-(54) represent the draining of a thin film down a dry wall. For example, equation (52) describes the draining of a thin film in the presence of a precursor film of height $\delta$. Moriarty and Schwartz [13] have considered

$$
f(y)=-1+\frac{1+\alpha}{y^{2}+\alpha}
$$

where $\alpha$ is the slip coefficient. This case is valid for dry wall coating where slip occurs in the vicinity of the contact line. This model was derived by Greenspan [6]. We can employ (24)-(26) to perform a reduction to second-order and proceed as indicated in this paper.

Acknowledgements- EM acknowledges support from the University Research Committee at the University of the Witwatersrand, Johannesburg, through the Friedel Sellschop award.

\section{REFERENCES}

[1] Bernis, F and Peletier, L. A., Two problems from draining flows involving third-order ordinary differential equations, SIAM J. Math. Anal., 27, 515-527, 1996.

[2] Baumann, G., Symmetry Analysis of Differential Equations With Mathematica, Springer-Verlag, Berlin, 2000.

[3] Bluman, G. W. and Kumei, S., Symmetries and differential equations, Springer-Verlag, New York, 1989.

[4] Duffy, B. R., Wilson, S. K., A third-order differential equation arising in thinfilm flows and relevant to Tanner's Law, Appl. Math. Lett., 10, 63-68, 1997.

[5] Ford, W. F., A third-order differential equation, SIAM Review, 34, 121-122, 1992.

[6] Greenspan, H. P., On the motion of a small viscous droplet that wets a surface, J. Fluid Mech., 84, 125-143, 1978.

[7] Head, A. K., LIE, a PC program for Lie analysis of differential equations, Computer Physics Communications, 77, 241-248, 1993.

[8] Ibragimov, N. H. and Mahomed, F. M., CRC Handbook of Lie Group Analysis of Differential Equations Vol. 3, CRC Press Inc., Boca Raton, Florida, Chp 8, 191-215, 1996. 
[9] Ibragimov, N. H., Elementary Lie group analysis and ordinary differential equations, J. Wiley and Sons, Chichester, 1999.

[10] Lambert, J. D., Numerical methods for ordinary differential systems: The initial value problem, J. Wiley and Sons, Chichester, 1991.

[11] E. Momoniat, Symmetries, first integrals and phase planes of a third-order ordinary differential equation from thin film flow, Mathematical and Computer Modelling, 49, 215-225, 2009.

[12] E. Momoniat, Numerical investigation of a third-order ODE from thin film flow, Accepted.

[13] Moriarty, J. A. and Schwartz, L. W., Effective slip in numerical calculations of moving-contact-line problems, J. Engng. Math., 26, 81-86, 1992.

[14] Myers, T. G., Thin films with high surface tension, SIAM Rev., 40, 441-462, 1998.

[15] Olver, P. J., Applications of Lie Groups to Differential Equations, SpringerVerlag, New York, 1986.

[16] Ovsiannikov, L. V., Group Analysis of Differential Equations, Academic Press, New York, 1982.

[17] Polyanin, A. D. and Zaitsev, V. F., Handbook of Exact Solutions for Ordinary Differential Equations, CRC Press Inc., Boca Raton, Florida, 1995.

[18] Tanner, L. H., The spreading of silicone oil drops on horizontal surfaces, J. Phys. D: Appl. Phys., 12, 1473-1484, 1979.

[19] Tuck, E. O. and Schwartz, L. W., Numerical and Asymptotic Study of Some Third-Order Ordinary Differential Equations Relevant to Draining and Coating Flows, S.I.A.M. Review, 32, 453-469, 1990.

[20] Sherring, J., Head, A. K. and Prince, G. E., Dimsym and LIE: symmetry determining packages, Mathematical and Computer Modelling, 25, 153-164, 1997.

[21] Troy, W. C., Solutions of third-order differential equations relevant to draining and coating flows, SIAM J. Math. Anal., 24, 155-171, 1993.

[22] Wolfram, S., The Mathematica Book, 3rd ed., Wolfram Media/Cambridge University Press, 1996. 